\title{
ROLE OF GINKGO BILOBA IN TINNITUS BASED ON TINNITUS HANDICAP INVENTORY
}

\begin{abstract}
Authors: Monica Manhas(1), Parmod Kalsotra (2), Aditiya Saraf *(3), Amit Manhas(4), Gopika Kalsotra (5) Authors Affiliations: (1)Assistant Professor, Department of Physiology, (2) Professor and Head, (3) Senior Resident, Department of ENT, (4) Consultant, Department of Anaesthesia, (5) Assistant Professor, Department of ENT, GMC Jammu
\end{abstract}

\section{ABSTRACT}

\section{AIMS AND OBJECTIVES}

To evaluate whether there is role of Ginkgo biloba in treatment of tinnitus or not, using Tinnitus Handicap Inventory

MATERIAL METHODS: The present prospective study, after approval by Institutional Ethics Committee, was conducted in Department of ENT, GMC Jammu from January 2019 to January 2020 on 90 patients with primary complaint of tinnitus. Inclusion criteria- Age: 20 years or above, Duration of tinnitus $>3$ months. Informed consent was taken and all patients were asked to complete the THI-Tinnitus Handicap Inventory. All patients were given $50 \mathrm{mg}$ of Ginkgo biloba standardized extract LI 1370 (containing 25\% flavonoids, $3 \%$ ginkgolides and 5\% bilobalides). Patients were advised to take three tablets daily for 12 weeks.

\section{RESULTS}

The mean pre-treatment THI score was 43.6 and mean post-treatment THI score (at 12 weeks) was 39.4; the mean change in THI score was 4.2, the result being statistically insignificant ( $p$-value= 0.43). Out of 90 patients, 51 reported unchanged severity of tinnitus, 27 patients reported improvement in severity of tinnitus and 12 patients reported worsening of condition.

\section{CONCLUSION}

According to our study, Ginkgo biloba has no significant therapeutic role in tinnitus. However, the aetiology of tinnitus is multifactorial and Ginkgo biloba could benefit patients with ischemic aetiology.

\section{KEYWORDS}

Ginkgo, biloba, handicap, inventory, tinnitus

\section{INTRODUCTION}

The term 'tinnitus' comes from a latin word 'tinnire' meaning 'to ring' and was introduced by Pliny. Tinnitus is an auditory perception due to altered spontaneous activity within auditory pathway, arising as a result of aberrant excitation or inhibition. The underlying mechanism for tinnitus can be : abnormal afferent excitation at cochlear level ( glutamate neuroexcitotoxicity, modulation of NMDA and non-NMDA receptors, calcium channel dysfunction ), efferent dysfunction ( reduction of GABA effect), alteration of spontaneous activity \& tonotopic reorganization, and, stress/psychological disorders. Tinnitus can be classified as subjective (heard only by the patient) or objective (heard by examiner as well). Subjective tinnitus means origin may be within external ear, middle ear, inner ear, VIII nerve or central nervous system. Objective tinnitus may be seen in vascular conditions (like AV shunts, carotid aneurysm, persistent stapedial artery, dehiscent jugular bulb), patulous eustachian tube, temporomandibular joint abnormality etc. [1]

Ginkgo biloba is an extract from the leaves of the maidenhair plant, with active substances including flavinoids (ginkgo-flavone glycosides) and terpenoids (ginkgo-flavone glycosides) and terpenoids (ginkgolides A,B,C,J and bilobalide). There are several proposed mechanism of actions of Ginkgo biloba- vasoregulatory effect promoting blood vessel, antagonism of platelet activating factor (PAF), changes in nerve cell metabolism, and, prevention of cell membrane 
damage by free radicals. Traditionally, Ginkgo biloba has been used in China for bronchitis and asthma. Now, its therapeutic use has been expanded to tinnitus.[2]

A wide range of other treatment modalities are available for tinnitus like pharmacological interventions (vasodilators, cortisone, antidepressants), hyperbaric oxygen therapy, cognitive behavior therapy, tinnitus masking, tinnitus retraining therapy, acupuncture etc. But none of these modalities can be declared satisfactory in all patients with tinnitus. Furthermore, Ginkgo biloba is a very commonly prescribed drug for tinnitus and there have been very few studies assessing the role of ginkgo biloba in tinnitus.

Hence ,with our study, we aim to fill this lacunae and evaluate whether there is role of Ginkgo biloba in treatment of tinnitus or not, using Tinnitus Handicap Inventory.

\section{MATERIAL AND METHODS}

The present prospective study, after approval by Institutional Ethics Committee, was conducted in Department of ENT, GMC Jammu from January 2019 to January 2020 on 90 patients with primary complaint of tinnitus.

Inclusion criteria- Age: 20 years or above, Duration of tinnitus $>3$ months

Exclusion criteria- History of middle ear disease or ear surgery, History of abnormal blood pressure, History of intake of anti-depressants or aspirin use, Pregnancy.

All 90 patients were asked relevant clinical history. All patients were subjected to general physical and systemic examination. Local ENT examination including otoscopy and tuning fork tests, was done on all patients.

Informed consent was taken and all patients were asked to complete the THI-Tinnitus Handicap Inventory (Newman CW et al. [3]). The first $\mathrm{THI}$ assessment was done prior to start of treatment, second at 4th week, third at 8th week and final assessment at 12th week of treatment. $\mathrm{THI}$ is a validated measure of tinnitus handicap and comprises of 25 questions. It is scored from 0 (slight handicap) to 100 (catastrophic handicap)

\begin{tabular}{|l|l|}
\hline $0-16$ & Slight/No handicap \\
\hline $18-36$ & Mild handicap \\
\hline $38-56$ & Moderate handicap \\
\hline $58-76$ & Severe handicap \\
\hline $78-100$ & Catastrophic handicap \\
\hline
\end{tabular}

All patients were given 50mg of Ginkgo biloba standardized extract LI 1370 (containing 25\% flavonoids, $3 \%$ ginkgolides and $5 \%$ bilobalides). Patients were advised to take three tablets daily for 12 weeks.

All data was entered in Microsoft Excel SpreadSheet and analysed and compared using the Statistical Package for Social Sciences (SPSS) software (version 21 for windows). Appropriate statistical analytical tests were applied ( $p$-value 0.05 or less considered significant).

\section{TINNITUS HANDICAP INVENTORY (Newman} CW et al. [3])

Following is the questionnaire asked to patients with tinnitus. The answers can be Yes (4), Sometimes (2) or No (0)

Tinnitus Handicap Inventory Questions

- Is it difficult to concentrate because of tinnitus

- Is it difficult to hear people

- Does it make you angry

- Does it make you feel confused

- Do you feel desperate due to tinnitus

- Do you complain a great deal about tinnitus

- Do you have trouble falling in sleep

- Do you feel you cannot escape tinnitus

- Does it interfere with your social activities

- Do you feel frustrated

- Do you feel you have a terrible disease

- Does tinnitus make it difficult to enjoy life

- Does it interfere with your job

- Do you feel irritable

- Is it difficult to read due to tinnitus

- Does it make you upset 
- Has it led to stress on family relationships

- Is it difficult to focus attention on other things

- Do you feel you have no control over tinnitus

- Do you feel tired

- Do you feel depressed

- Do you feel anxious

- Do you feel you can no longer cope with tinnitus

- Does it get worse with stress

- Does it make you insecure

Sum of all the responses is the THI Score

\begin{tabular}{|c|c|c|c|c|c|}
\hline \multicolumn{7}{|c|}{ THI Grading based on THI score } \\
\hline Grade & THI Score & Grade & THI Score & Grade & THI Score \\
\hline Grade 1 & $0-16$ & Grade 3 & $38-56$ & Grade 5 & $78-100$ \\
\hline Grade 2 & $18-36$ & Grade 4 & $58-76$ & & \\
\hline
\end{tabular}

\section{RESULTS}

The mean age of presentation was $59.16 \pm 6.55$ years, with maximum number of patients in the age group of $51-60$ years (51.1\%). Out of 90 patients, 61 were males (67.7\%) and 29 were females (32.2\%), with male to female ratio being 2.1:1.

The mean duration of tinnitus was about $4.92 \pm$ 1.53 years. Out of 90 patients, 78 patients had bilateral tinnitus (86.6\%) and 12 patients (13.3\%) had unilateral tinnitus. Tinnitus was constant in 65 patients (72.2\%) and intermittent in 25 patients (27.7\%).

Out of 90 patients, 38 patients had moderate handicap on $\mathrm{THI}$ scoring grade (42.2\%), 33 patients had mild handicap (36.6\%), 17 patients had slight handicap (18.8\%) and 2 patients had severe handicap (2.2\%)

\begin{tabular}{|c|c|}
\hline Handicap on THI & Number of Patients (\%) \\
\hline Slight & $17(18.8 \%)$ \\
\hline Mild & $33(36.6 \%)$ \\
\hline Moderate & $38(42.2 \%)$ \\
\hline Severe & $2(2.2 \%)$ \\
\hline Catastrophic & Nil \\
\hline
\end{tabular}

The mean pre-treatment THI score was 43.6 and mean post-treatment $\mathrm{THI}$ score (at 12 weeks) was 39.4; the mean change in THI score was 4.2, the result being statistically insignificant $(p$-value $=0.43)$. Out of 90 patients, 51 reported unchanged severity of tinnitus, 27 patients reported improvement in severity of tinnitus and 12 patients reported worsening of condition.

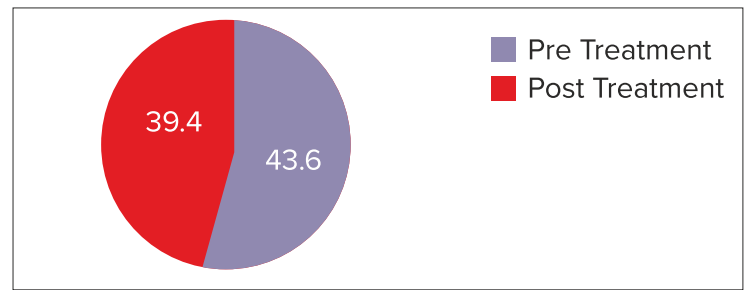

\section{DISCUSSION}

Tinnitus, a form of auditory dysfunction, is a common condition that is recognized as a major health issue by about $0.5 \%$ of world's population. Tinnitus is considered to be multifactorial in its aetiology, hence a single effective treatment for managing it, is highly unlikely. Tinnitus is a form of sensory epilepsy in which brain generates an abnormal memory of a sensation which is sustained long after the sensory receptors have been damaged, which appears to be associated with burst firing due to a decrease in GABAergic inhibition and an increase in Glutamatergic excitation.[4]

Due to scarcity of animal studies and very few clinical trials, medical treatment options for tinnitus are highly limited and efficacy of various drugs used for tinnitus is still unreliable. Due to association with epilepsy, anti-epileptic drugs have been used. Sedative-hypnotics have also been used in managing tinnitus. However, usage of these class of drugs for tinnitus is highly controversial because of their adverse side effects. Intratympanic gentamycin (to inactivate dysfunctional cochlear hair cells) and intratympanic steroids ( anti-inflammatory action) have been also used for treating tinnitus.[5]

Ginkgo biloba (Ginkgoaceae) is an ancient Chinese tree which has been cultivated for its health promoting properties for thousands of years. It has been widely used for peripheral vascular disease and cerebral insufficiency. Role of ginkgo in alleviating tinnitus is still under research. We, with this study, aimed to study role 
of ginkgo biloba in tinnitus.

In our study, the mean age of presentation was about $59.16 \pm 6.55$ years. This observation was comparable to studies conducted by Drew $S$ et al. (2001) [6] and Rejali D et al. (2004) [7] . The increased prevalence of tinnitus in elderly population may be due to associated metabolic, neurologica, otologic or cardiovascular comorbidity. In our study, there was male preponderance, which is comparable to study conducted by Han SS et al (2012) [8] . However, Seydel C et al. [9] in their study showed female preponderance. The reason for male preponderance may be due to the fact that perception of severity is closely related to personality traits like neuroticism. The mean duration of tinnitus in our study was about $4.92 \pm$ 1.53 years. This observation was comparable to studies conducted by Ernst E at el.(1999) [10] and Morgenstern et al. (1997) [11].

In our study, we used Tinnitus Handicap Inventory to measure the outcome of Ginkgo biloba therapy, which is consistent with studies by Rejali D et al. (2004) [7] and Han SS et al. (2004) [8]. However, Drew S et al. (2001) [6] used Tinnitus disability score and Holgers KM et al.(2003) [9] used Visual analogue score to measure outcome of ginkgo biloba extract treatment. The mean pre-treatment THI score was 43.6 and mean post-treatment THI score was 39.4; the mean change in $\mathrm{THI}$ score being 4.2 , the result being statistically insignificant ( $p$ value $=0.43$ ). This finding was consistent with studies conducted by Hilton and Stuart (2004) [11] \& Meehan et al. (2004) [12], who also in their studies showed insignificant improvement in THI score. However, Jastreboff et al. (1997) [13] in their study showed statistically significant improvement in THI score post treatment ( $p$ value less than 0.05). The reason for improvement in tinnitus score after ginkgo biloba treatment could be due to the fact that ginkgo biloba has anti-ischemic and vasodilatory properties, thus alleviating one of the important cause of tinnitus, that is, deficiency of blood supply to inner ear. Also, improved cognitive functioning with ginkgo biloba allows habituation to tinnitus.

\section{CONCLUSION}

The misconception about ginkgo biloba being an effective drug in treating tinnitus can deviate clinicians from other more reliable treatment options. According to our study, Ginkgo biloba has no significant therapeutic role in tinnitus. However, the aetiology of tinnitus is multifactorial and Ginkgo biloba could benefit patients with ischemic aetiology.

\section{CONFLICT OF INTEREST: NIL FUNDING SPONSORSHIP: NIL}

\section{BIBLIOGRAPHY}

1) Ceranic B, Luxon LM. Tinnitus and other dysacuses. In: Gleeson M, Browning GG, Burton MJ et al, editors. Scott Brown's Otorhinolaryngology, Head and neck surgery.Great Britain:Edward Arnold; 2018.p.3594-362

2) Macleman $K$, Darlington $C L$, Smith PF. The CNS effects of Gingo Biloba extracts and gingolide $B$. Progress in Neurobiology 2002;67:236-58

3) Newman CW, Jacobson GP, Spitzer JB. Development of the Tinnitus Handicap Inventory. Arch Otolaryngol Head and Neck Surg 1996;122(2):143-8

4) Eggermont JJ, Roberts LE. The neuroscience of tinnitus. Trends Neuroscience 2004;27:676-82

5) Simpson J, Davies WE. Recent advances in the pharmacological treatment of tinnitus. Trends in Pharmacology Science 1999;20:128

6) Drew S, Davies E. Effectiveness of Ginkgo Biloba in treating tinnitus: double blind, placebo controlled trial BMJ 2001;322:1-6

7) Rejali $D$, Sivakumar A, Balaji N. Ginkgo Biloba does not benefit patients with tinnitus: a randomized placebo-controlled double blind trial and meta-analysis of randomized trials. Clin Otolaryngol Allied Sci 2004;29:226-31

8) Han SS, Nam EC, Won JY et al. Clonazepam quiets tinnitus: a randomized crossover study 
with Ginkgo Biloba. J Neurol Neurosurg Psychiatry 2012;83:821-27

9) Holgers KM, Barrenas ML, Svedlund J et al. Clinical Evaluation of Tinnitus: A Review 2003;2:101-6

10) Ernst E, Stevinson C. Ginkgo Biloba for tinnitus: a review. Clin Otolaryngol 1999;24:164-7

11) Hilton M, Stuart E. Ginkgo biloba for tinnitus. Cochrane Database Syst Rev 2004;2:CD003852

12) Meehan T, Eisenhut M, Stephens D. A review of alternative treatments for tinnitus. Audiological Medicine 2004:2;74-82

13) Jastreboff PJ, Zhou S, Jastreboff MM et al. Attenuation of salicylate induced tinnitus by ginkgo biloba extract in rats. Audiol Neurootol 1997;2:197-212

\section{*Corresponding Author:}

Dr Adtitya Saraf

Senior Resident

Department of ENT, GMC Jammu

Email ID: rksaraf56@gmail.com 